\title{
Stellar Structure and the Origin of Stellar Energy.*
}

By Prof. E. A. Mrune, F.R.S.

$\mathrm{P}$ ERHAPS the most striking general characteristic of the stars is that they can be divided into two groups of widely differing densities. In the first group, which comprises the majority of the known stars, the densities are of 'terrestrial' order of magnitude; that is to say, their mean densities are of the order of the known densities of gases, liquids, and solids. They range from onemillionth of that of water to ten or, in rare cases, perhaps fifty times that of water. In the second group the densities are of the order of 100,000 times that of water. Of the second group, the 'white dwarfs', only a few examples are known, but they are all near-by stars, and it is generally agreed that they must be of very frequent occurrence in Nature, though difficult of discovery owing to their faintness. Whether stars exist of intermediate density remains for future observation. The possibility of the existence of matter in this dense state offers no difficulty. As pointed out by Eddington, we simply have to suppose the atoms ionised down to free electrons and bare nuclei. At these high densities the matter will form a degenerate gas, as first pointed out by R. H. Fowler. But this leaves entirely unsolved the question of why, under stellar conditions, matter sometimes takes up the 'normal' density and sometimes the high density. Owing to the probable great frequency of occurrence of dense stars, it might reasonably be asked of any theory of stellar constitution that it should account for dense stars in an unforeed way.

There are two main theories of stellar structure at the present moment. That of Sir James Jeans accounts for the existence of giants, dwarfs, and white dwarfs, but only at the cost of ad hoc hypotheses quite outside physics. It assumes stars to contain atoms of atomic weight higher than that observed on earth, and it assumes them to be relentlessly disappearing in the form of radiation; it appeals to discontinuous changes of state consequent on successive ionisations, for which there is little warrant. I think it is true to say that the majority of astronomers do not accept this theory.

The theory of Sir Arthur Eddington does not claim to account for the observed division of stars into dense stars and stars of ordinary density; nor does it establish the division of ordinary stars into giants and dwarfs. On the other hand, it claims to establish what is known as the mass-luminosity law from considerations of equilibrium only, that is, without introducing anything connected with the physics of the generation of energy. It claims to show that the observed fact that the brighter stars are the more massive can be deduced from the conditions expressing that the star is in a steady state, mechanically and thermally. It does this by making the hypothesis that the stars (giants and ordinary dwarfs) consist of perfect gas. Closer

- Substance of lectures delivered at the Royal Institution on Dec. 2 and Dec. $9,1930$. consideration of the actual formulæ used by the theory shows that it scarcely bears out the claims made for it by its originator. The "formula for the luminosity' of a star makes the luminosity very nearly proportional to its effective temperature, and so the so-called proof of the mass-luminosity law involves a semi-empirical element, namely, an appeal to the observed effective temperatures of the stars, for the observed values of which the theory fails to account. Another difficulty encountered by the theory is that it makes the interiors of the more luminous (giant) stars cooler than those of the fainter stars, and it makes the interiors of both too cool for the temperature to have any appreciable influence on the rate of generation of energy, by stimulating, for example, the production of radioactive elements or the conversion of matter to radiation.

The claim to establish the mass-luminosity law from mere equilibrium considerations cannot, however, be sustained for a moment. We may regard a star in a steady state as a system provided with an internal heating apparatus (the source of energy). It adjusts itself-state of aggregation, density distribution, temperature distribution-until the surface emission equals the internal generation of energy $L$. But provided the luminosity $L$ is not too large (in order that the mass shall not burst under radiation pressure), it is clear that a given mass $M$ can adjust itself to suit any arbitrary value of $L$. If, starting with one steady state, we then alter $L$ (upwards or downwards) by altering the rate of supply of energy, the star will simply heat up or cool down until the surface emission is equal to the new volume of $L$-precisely like an electric fire. $L$ and $M$ are thus independent variables so far as steady-state considerations are concerned. The fact that $L$ and $M$ show a degree of correlation in Nature must be connected with facts of an altogether different order, namely, with the physics of energy-generation. It is essential to recognise the difference between the formal independence of $L$ and $M$ as regards steady-state considerations and the observed correlation of $L$ with $M$ in Nature. The observed mass-luminosity law must depend on the circumstance that in some way the more massive star contrives to provide itself with a stronger set of sources. The claim to establish the mass-luminosity law from equilibrium considerations only appears to me a philosophical blunder. Further, it is unphilosophical to assume the interior of a gas to be a perfect gas ; either knowledge of the interior is for ever unattainable or we should be able to infer it from the observable outer layers.

When we dispense with the perfect gas hypothesis and at the same time recognise the independence of $L$ and $M$ as regards steady-state considerations, it is found that a rational analysis of stellar structure automatically accounts for the existence of dense stars without special hypothesis. Further, it shows, as common sense would lead us to expect, that the more luminous stars must have 
the hotter interiors. Here the temperatures are found to range up to $10^{10}$ degrees or higher, depending on luminosity - a temperature sufficient to stimulate the conversion of matter into radiation. In addition, it shows that the central regions of stars must be very dense, ranging up to $10^{7}$ grams $\mathrm{cm}^{-3}$ or higher. Thus the difficulties met by earlier theories fall away as soon as the ground is cleared philosophically.

The foregoing ideas suggest the following as the fundamental problems of stellar structure: (1) What are the configurations of equilibrium of a prescribed mass $M$ as its luminosity $L$ ranges from 0 upwards, $M$ remaining constant ? (2) What is the effective temperature $T$ associated with a given pair $(M, L)$ in a steady state? (3) What is the value of $L$ which will actually occur for the physical conditions disclosed by the answer to problem (1) ?

We observe that the outer parts of a star are gaseous. Consequently we can solve the problem of the state of any actual star by integrating the equations of equilibrium from the boundary inwards; we are entitled to assume the gas laws to go on holding until we find that the conditions are incompatible with them. We then change to a new equation of state, and carry on as before. We change our equation of state as often as may be necessary until we arrive at the centre.

The answer to the first of the problems formulated above has been worked out, for certain types of source-distribution and opacity, by the method of inward integration. The results are sufficiently alike to be taken as affording insight into the nature of stellar structure in general, and are as follows. For a given mass $M$, of prescribed opacity, there exist two critical luminosities $L_{1}$ and $L_{0} \quad\left(L_{1}>L_{0}\right)$ such that for $L>L_{1}$ no configurations of equili-

brium exist; for $L_{1}>L>L_{0}$ the density and temperature increase very rapidly as the centre is approached $\left(T \propto r^{-1}\left(\log \frac{\text { const. }}{r}\right)^{-\frac{1}{2}}\right)$, so that in the centre there is a region of very high temperatures and densities where the gas laws are violated; for $L=L_{0}$ a diffuse perfect gas configuration is possible ; for $L_{0}>L>0$ the only perfect gas configuration is a hollow shell provided with an internal, rigid supporting surface of spherical shape. Since in Nature no internal supporting surface is provided, to find the actual configuration when $L_{0}>L>0$ we construct the artificially supported hollow configuration and then remove the supporting surface. The mass must collapse, and collapse will proceed until a steady-state is attained in which, except for a gaseous outer fringe, the gas laws are violated. Such configurations may be termed 'collapsed'. Configurations for which $L_{1}>L>L_{0}$ may be termed
' centrally-condensed'. The physical origin of the different types of configuration is simply the varying effect of light-pressure. For $L=L_{0}$ the light-pressure due to $L$ is just sufficient to distend the star against its self-gravity and maintain it in the form of a perfect gas. For $L_{1}>L>L_{0}$ light-pressure is so high that for equilibrium to be maintained gravity at any given distance from the centre must be assisted by concentrating as much matter as possible inside the sphere in question; when this process is carried out for all spheres, we get a central condensation. For $L_{0}>L>0$, light-pressure due to $L$ is so low that the mass cannot support itself against its own weight in the form of perfect gas, and collapse sets in until the gas-laws are disobeyed. The diffuse configurations $L=L_{0}$ are unstable with respect to small changes of $L$.
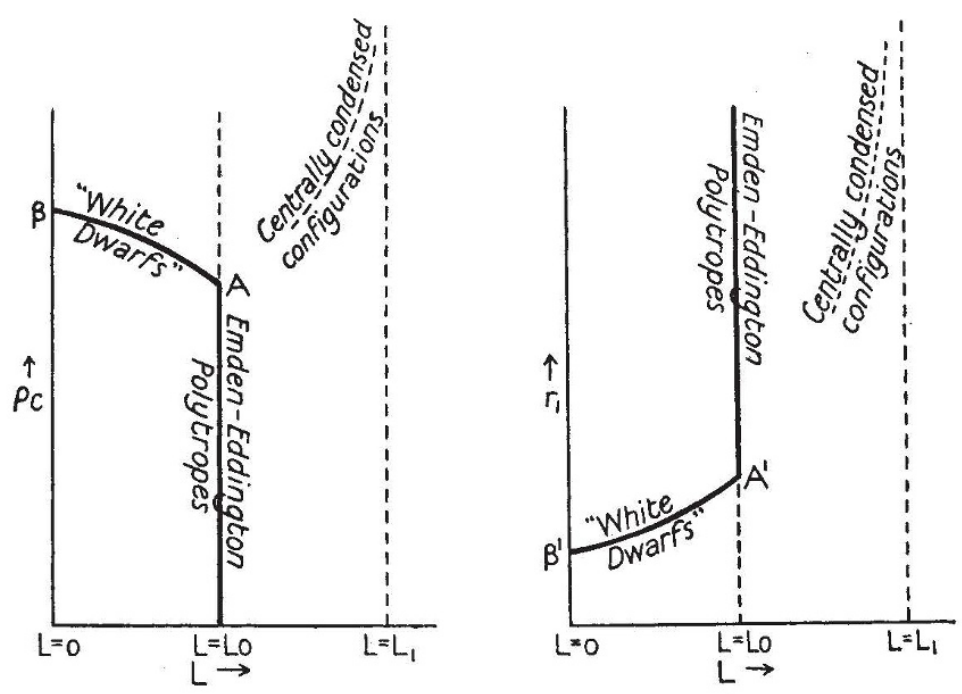

No. 3192, VoL. 127]

For collapsed or centrally-condensed configurations the centre will be occupied by a gas in a degenerate state. When the mean densities or effective temperatures of collapsed configurations are calculated, using the Fermi-Dirac statistics for the degenerate gas, they are found to agree with the observed order of magnitude for white dwarfs. Thus, collapsed configurations may be identified with white dwarfs. A white dwarf is thus a dense star simply because its luminosity is too low, and its light-pressure accordingly too low, for it to support its own mass against its own gravity. From another point of view the calculation affords an observational verification of the numerical value of the 'degenerate gas constant' the co. efficient $K$ in the degenerate gas law $p=K \rho^{5}$, and so a check on the Fermi-Dirac statistics.

If collapsed configurations may be identified with white dwarfs, centrally-condensed configurations 
may be provisionally identified with ordinary giants and dwarfs, though the full determination of the properties of centrally-condensed configurations awaits the construction of certain tables. Centrallycondensed configurations appear to have the properties that as $L$ decreases from $L_{1}$ to $L_{0}$ the effective temperature rises to a maximum and then decreases again. This would correspond to the observed division into giants and dwarfs. I give this deduction with some caution, as it is not yet demonstrated rigorously in the absence of the tables above mentioned.

A point not yet settled is the question of the continuity of the series of centrally-condensed configurations with the collapsed configurations (Figs. 1 and 2). There are indications that as $L$ passes through $L_{0}$ from above to below, the external radius of the configuration may decrease discontinuossly, the gaseous envelope collapsing on to the dense core. If this is confirmed, it would follow that a star, when its steady-state luminosity $L$ falls through a certain critical value (depending on its mass), exhibits the phenomena of a nova or temporary star. For it would have to disengage a large amount of gravitational potential energy in a short time, so that the actual emission would undergo a temporary increase, falling again to a value just below its previous value. It would be highly interesting to have observational data as to the densities of a nova before and after the outburst. The early-type spectrum of the later stage of a nova may indeed be taken to indicate a high effective temperature, and so a small radius and high density, in accordance with our prediction.

The important point about all the foregoing analysis is that it involves at no stage any special properties of matter or special assumptions. The observed features of the stars are thus found to depend only on the most general properties of matter in association with light-pressure.

A question logically distinct from these is the origin of stellar energy. Here we require to know something of the physics of energy-generation. The following suggestions are frankly of a speculative character. Let us assume, in accordance with a hypothesis first made by Jeans (not his later hypothesis of super-radioactive atoms), that protons and electrons can unite to form radiation. Then thermodynamic considerations show that the process must be reversible-photons can generate matter. We know that matter at ordinary tem. peratures is stable. Hence we may postulate the existence of a critical temperature above which the process can go on in either direction. Suppose this critical temperature has been passed at $10^{11}$ degrees. Calculation then shows that at $10^{11}$ degrees almost the whole of the mass in an enclosure would be in the form of radiation; and further, that lowering of the temperature of the enclosure would result in more of the surviving matter present disappearing in the form of radiation. The process is in fact the thermorlynamic opposite of evaporation: steam condenses to water with emission of energy, and the process is accordingly encouraged by cooling; matter 'evaporates' (to radiation) with emission of energy, and the process is encouraged by cooling. Now, the centre of a star is a sort of thermodynamic enclosure with a slight leak. It follows that if (as the steady-state theory indicates) the central region of a fairly luminous star is at a temperature of $10^{11}$ degrees and a high density, then this central region is effectively a reservoir of very dense radiant energy, with a mere sprinkling of ordinary matter present. Natural cooling of this reservoir provides the star's emission to space, and the reservoir is itself maintained by the conversion of matter into radiation inside it and on its confines.

Calculations based on this idea are consistent with the usually accepted evolutionary time scale, and predict a rate of 'generation' of energy $\epsilon$ per gram of the right order, namely, $\epsilon=-\frac{4 c^{2}}{T} \frac{d T}{d t}$ throughout the mass of the core, where $-d T / d t$ is the rate of cooling. The rate of loss of mass is given by the usual formula $\frac{d M}{d t}=-\frac{L}{c^{2}}$. By combination of these formulæ it is found possible in principle to establish a relation linking $M$ with $T_{c}$ (the central temperature); this is the additional relation which, by expression of $T_{c}$ in terms of $L$ and $M$ by means of the steady-state theory, must lead in due course to a mass-luminosity correlation. Whether it agrees with the observed mass luminosity law remains for future investigation, but it is a final satisfaction that, after first considering $L$ and $M$ as independent variables, we are able to use the equilibrium configurations thus disclosed to arrive in outline at a solution of the problem of the actual correlation of mass and luminosity in Nature. It is to be noted that the star's generation of energy is naturally non-explosive, for it is simply a consequence of the natural tendency of the star to cool. The star behaves, in fact, simply like a freely cooling body containing a central region of very high specific heat-namely, a pool of intense radiant energy, which is gradually drained away though partially reinforced by the conversion of matter. From this point of view, it is not that a star descends an evolutionary path because its rate of generation of energy slackens; it is rather that the act of evolving and the act of radiating energy are identical.

'These suggestions as to the origin of stellar energy and the mode of stellar evolution are not to be pressed. They are to be sharply distinguished from the steady-state theory, which by the rational process of proceeding from the known stellar exterior step by step into the unknown interior indicates an inevitable series of configurations which correspond to the observed bifurcation of celestial objects into 'ordinary' stars and 'dense' stars.

NoTE.-The fundamental result of the rational method of analysis of stellar structure described in the foregoing article is the division of configurations into two types, the 'collapsed' and the "centrally-condensed'. The existence of these two types can be demonstrated without complicated mathematics by the following argument. Let $r_{1}$ (Continued on p. 27.) 
be the radius of a configuration, arbitrarily assigned beforehand. Let us endeavour to construct a gaseous configuration with this radius. If such a configuration be capable of being constructed, let us in imagination take a journey inwards to the centre, starting from the boundary. Let $M$ be the total mass, $M(r)$ the 'surviving' mass left inside the sphere of radius $r$ when we have reached the distance $r$ from the centre. Then $M-M(r)$ is the mass already traversed. Consider now the influence of light-pressure. If $L$ is large, light-pressure will be large and will balance an appreciable fraction of gravity, and accordingly the density-gradient will be small. But if $L$ is small, light-pressure will be small, and the density-gradient will be large. Thus, when $L$ is large, we shall have traversed a smaller mass $M-M(r)$ in the shell between $r_{1}$ and $r$ than when $L$ is small. Consequently, when $L$ is large, $M(r)$ will be larger than when $L$ is small.
In other words, as we journey inwards, when $L$ is small we ' consume our mass' faster than when $L$ is large. If $L$ is sufficiently small, we may have consumed our whole mass $M$ before we arrive at the centre ; in that case the only configuration of radius $r_{1}$ and mass $M$ is a hollow shell internally supported by a rigid spherical surface. If $L$ is sufficiently large, we shall, however, tend to have an appreciable mass $M(r)$ surviving unconsumed however near we approach the centre, and this surviving mass $M(r)$ can only be packed inside $r$ at the cost of high density with violation of the gas laws. Thus these configurations for large $L$ must be centrally condensed. For small $L$, on the other hand, no configurations of radius $r_{1}$ and mass $M$, unsupported, can exist, and the actual configurations must be 'collapsed' ones. 'Collapsed' configurations prove to be much more nearly homogeneous than 'centrally-condensed' ones.

\section{Scientific Centenaries in I931.}

W ITH the forthcoming celebrations in London in September, first of the centenary of the discovery by Faraday of electromagnetic induction and then of the centenary of the British Association, the year 1931 promises to be one of outstanding interest to men of science, especially as these events will be preceded by the Second International Congress of the History of Science and Technology, also to be held in London. At Cambridge, too, a committee of distinguished men has been appointed in connexion with the centenary celebrations of the birth of Maxwell (1831-1879). Among other famous physicists who were born the same year as Maxwell were P. G. Tait (1831$1901)$; D. E. Hughes (1831-1900); Sir Francis Bolton (1831-1887), a founder of the Institution of Electrical Engineers; S. H. Burbury (1831-1911); and Johannes Bosscha (1831-1911), once the acknowledged leader of Dutch physicists; while a hundred years ago died J. T. Seebeck (1770-1831), the discoverer of thermo-electricity, and Henry Foster (1796-1831), the Copley Medallist of the Royal Society in 1827. Two hundred years ago, on Oct. 10, 1731, Cavendish was born at Nice.

Among the anmiversaries of 1931 of great interest to mathematicians is that of the tercentenary of the death of Henry Briggs (1561-1631), the first Gresham professor of geometry, and from 1620 Savilian professor at Oxford, an appointment in which he succeeded Savile himself. The first improver of logarithms after Napier, Briggs wrote two great works, his "Arithmetica Logarithmica" and "Trigonometria Britannica". At his death Briggs was buried beside Savile in the chapel of Merton College. Another well-known name is that of Brook Taylor (1685-173I), " one of the most able British mathematicians after Newton", and secretary to the Royal Society. Baron Maseres (1731-1824), the mathematician and judge, was born the year Taylor died. Maseres, Taylor, Briggs, Burbury, Cavendish, and Tait, like Maxwell, were all graduates of Cambridge; while last century, few Cambridge mathematicians had a higher reputation than E. J. Routh (1831-1907), who shared the Smith prize with Maxwell. The year 1831 also saw the death of the remarkable French woman mathematician, Sophie Germain (1776-1831).

Of the names of astronomers recalled by the passing of a century, mention may be made of Jean Louis Pons (1761-1831), the discoverer of 37 comets; of the Rev. F. Fallows (1789-1831), the first Royal Astronomer at the Cape of Good Hope, where he died; of Edward Stone (18311897), who was the predecessor of Gill at the Cape; and Bredichin (1831-1904), the successor of Otto Struve at Pulkova Observatory. A very notable achievement in astronomy is recalled by the tercentenary of the observation by the philosopher Gassendi, on Nov. 7, 1631, of the transit of Mercury across the sun's disc. The occurrence had been predicted by Kepler, but Gassendi alone among his contemporaries appears to have made adequate arrangements for observing the transit, and happily his foresight and ingenuity were amply rewarded by his success.

Other distinguished men of science born a hundred years ago include the American agricultural chemist, Samuel William Johnson, whose two books, "How Crops Grow", 1868, and "How Crops Feed", 1870, were translated into French, German, Russian, Italian, and Japanese; the German chemist, Hans Heinrich Landolt (18311910), who was spoken of in the Journal of the Chemical Society as "the patriarch of physical chemistry"; and the British chemists, Matthiessen (1831-1870), Northcote (1831-1869), Bloxam (1831-1887), and Atkinson (1831-1900), all of whom enriched chemical literature. The year 1831 also saw the birth of the great Austrian geologist, Eduard Suess (1831-1914), Copley Medallist of the Royal Society in 1903, whose name is now to be seen on a tablet affixed to 4 Duncan Terrace, Islington, where he was born; of Carl Albert Oppel (1831-1865), the eminent German palæontologist; of Othniel Charles Marsh (1831-1899), long in charge of the division of vertebrate palæonto- 